\title{
Factorisation of germ-like series
}

\author{
SONIA L'INNOCENTE \\ VINCENZO MANTOVA
}

\begin{abstract}
A classical tool in the study of real closed fields are the fields $K((G))$ of generalised power series (ie, formal sums with well-ordered support) with coefficients in a field $K$ of characteristic 0 and exponents in an ordered abelian group $G$. A fundamental result of Berarducci ensures the existence of irreducible series in the subring $K\left(\left(G^{\leq 0}\right)\right)$ of $K((G))$ consisting of the generalised power series with non-positive exponents.

It is an open question whether the factorisations of a series in such subring have common refinements, and whether the factorisation becomes unique after taking the quotient by the ideal generated by the non-constant monomials. In this paper, we provide a new class of irreducibles and prove some further cases of uniqueness of the factorisation.
\end{abstract}

2010 Mathematics Subject Classification 13F25 (primary); 03E10, 12J15, 06F25 (secondary)

Keywords: generalised power series, unique factorisation, order-value

\section{Introduction}

If $K$ is a field and $G$ an additive abelian ordered group, a formal series with coefficients in $K$ and exponents in $G$ is a formal sum $a=\sum_{\gamma} a_{\gamma} t^{\gamma}$, where $a_{\gamma} \in K$ and $\gamma \in G$. We call support of $a$ the set $S_{a}:=\left\{\gamma \in G: a_{\gamma} \neq 0\right\}$. A formal series $a$ is said to be a generalised power series if its support $S_{a}$ is well-ordered. The collection of all generalised power series, denoted by $K((G))$, is a field with respect to the obvious operations + and $\cdot$ defined for ordinary power series (see Hahn [5]).

When $K$ is ordered, then $K((G))$ has a natural order as well, obtained by stipulating that $0<t^{\gamma}<a$ for any $\gamma \in G^{>0}$ and for any positive element $a$ of the field $K$. Moreover, if $K$ is real closed and $G$ is divisible, then $K((G))$ is real closed. Conversely, any ordered field can be represented as a subfield of some $\mathbb{R}((G))$ (Gleyzal [4]).

For these reasons, the field $K((G))$ is a valuable tool for the study of real closed fields. One can use them to prove, for instance, that every real closed field $R$ has an integer part 
(ie, a subring $Z$ such that for all $x \in R$ there exists a unique integer part $\lfloor x\rfloor \in Z$ of $x$ such that $\lfloor x\rfloor \leq x<\lfloor x\rfloor+1$ (Mourgues and Ressayre [6]). For example, $\mathbb{Z}+\mathbb{R}\left(\left(G^{<0}\right)\right.$ ) is an integer part of $\mathbb{R}((G))$, where $\mathbb{R}\left(\left(G^{<0}\right)\right)$ is the subring of the series with the support contained in the negative part $G^{<0}$ of the group $G$.

The ring $\mathbb{Z}+\mathbb{R}\left(\left(G^{<0}\right)\right)$ has a non-trivial arithmetic behaviour, some of which is already visible in $\mathbb{R}+\mathbb{R}\left(\left(G^{<0}\right)\right)=\mathbb{R}\left(\left(G^{\leq 0}\right)\right)$. When $G$ is divisible, the ring $K\left(\left(G^{<0}\right)\right)$ is non-noetherian, as for instance we have $t^{-1}=t^{-\frac{1}{2}} \cdot t^{-\frac{1}{2}}=t^{-\frac{1}{4}} \cdot t^{-\frac{1}{4}} \cdot t^{-\frac{1}{4}} \cdot t^{-\frac{1}{4}}=\ldots$ However, Berarducci [1] proved that $K\left(\left(G^{\leq 0}\right)\right)$, when $\mathbb{Q} \subseteq G$, contains irreducible series, such as $1+\sum_{n} t^{-\frac{1}{n}}$, answering a question of Conway [3]; in fact, his result implies that $1+\sum_{n} t^{-\frac{1}{n}}$ is irreducible in the ring of omnific integers, the natural integer part of surreal numbers, which are also of the form $\mathbb{Z}+\mathbb{R}\left(\left(G^{<0}\right)\right)$ for a suitable $G$.

In order to state Berarducci's result, let the order type ot $(a)$ of a power series $a \in$ $K\left(\left(G^{\leq 0}\right)\right)$ be the ordinal number representing the order type of its support $S_{a}$. Moreover, let $J$ be the ideal of the series that are divisible by $t^{\gamma}$ for some $\gamma \in G^{<0}$ (as noted before for $\gamma=-1$, such series cannot be factored into irreducibles when $G$ is divisible, since $t^{\gamma}=t^{\frac{\gamma}{2}} t^{\frac{\gamma}{2}}=\ldots$ ).

Theorem 1.1 (Berarducci [1, Theorem 10.5]) If $a \in K\left(\left(\mathbb{R}^{\leq 0}\right)\right) \backslash J$ (equivalently, $a \in K\left(\left(\mathbb{R}^{\leq 0}\right)\right)$ not divisible by $t^{\gamma}$ for any $\left.\gamma<0\right)$ has order type $\omega^{\omega^{\alpha}}$ for some ordinal $\alpha$, then both $a$ and $a+1$ are irreducible.

This result was obtained by constructing a function resembling a valuation but taking values into ordinal numbers.

Definition 1.2 ([1, Def. 5.2]) For $a \in K\left(\left(G^{\leq 0}\right)\right)$, the order-value $v_{J}(a)$ of $a$ is:

(1) if $a \in J$, then $v_{J}(a):=0$;

(2) if $a \in J+K$ and $a \notin J$, then $v_{J}(a):=1$;

(3) if $a \notin J+K$, then $v_{J}(a):=\min \left\{\operatorname{ot}\left(a^{\prime}\right): a-a^{\prime} \in J+K\right\}$.

The difficult key result of [1] is that for $G=\mathbb{R}$ the function $v_{J}$ is multiplicative.

Theorem 1.3 ([1, Theorem 9.7]) For all $a, b \in K((\mathbb{R} \leq 0))$ we have $v_{J}(a b)=v_{J}(a) \odot$ $v_{J}(b)$ (where $\odot$ is Hessenberg's natural product on ordinal numbers).

This immediately implies, for instance, that the ideal $J$ is prime, so the quotient ring of germs $K((\mathbb{R} \leq 0)) / J$ is an integral domain, and also each element admits a factorisation 
into irreducibles (in fact, $J$ is prime for arbitrary choices of $G$, see Pitteloud [8]; for further extensions to arbitrary groups $G$, see Biljakovic, Kochetov and Kuhlmann [2]). The above comments and theorems support and motivate the following conjectures. If $a=b_{1} \cdot \ldots \cdot b_{n}$ is a factorisation of a series $a$, possibly with some reducible factors, a refinement is another factorisation of $a$ obtained by replacing each $b_{i}$ with a further factorisation of $b_{i}$. More formally, a refinement is a factorisation $a=c_{1} \cdot \ldots \cdot c_{m}$ such that, up to reordering $c_{1}, \ldots, c_{m}, b_{i}=k_{i} \cdot c_{m_{i}+1} \cdot \ldots \cdot c_{m_{i+1}}$ for some constants $k_{i} \in K^{*}$ and some natural numbers $0=m_{1} \leq \cdots \leq m_{n+1}=m$.

Conjecture 1.4 (Conway [3]) For every non-zero series $a \in K\left(\left(\mathbb{R}^{\leq 0}\right)\right)$, any two factorisations of a admit common refinements.

For instance, it is easy to verify that for all $\gamma<0$, any two factorisations of $t^{\gamma}$ admit a common refinement. Similarly, any polynomial in $t^{\gamma}$ with coefficients in $K$ has infinitely many factorisations, but again any two of them admit a common refinement.

Conjecture 1.5 (Berarducci [1]) Every non-zero germ in $K((\mathbb{R} \leq 0)) / J$ admits a unique factorisation into irreducibles.

Berarducci's work was partially strengthened by Pitteloud [7], who proved that any (irreducible) series in $K\left(\left(\mathbb{R}^{\leq 0}\right)\right)$ of order type $\omega$ or $\omega+1$ and order-value $\omega$ is actually prime.

Adapting Pitteloud's technique, we shall prove that the germs of order-value $\omega$ are prime in $K\left(\left(\mathbb{R}^{\leq 0}\right)\right) / J$; in particular, the germs of order-value at most $\omega^{3}$ admit a unique factorisation into irreducibles, supporting Berarducci's conjecture.

Theorems 3.3-3.4 All germs in $K\left(\left(\mathbb{R}^{\leq 0}\right)\right) / J$ of order-value $\omega$ are prime. Every non-zero germ in $K\left(\left(\mathbb{R}^{\leq 0}\right)\right) / J$ of order-value $\leq \omega^{3}$ admits a unique factorisation into irreducibles.

Moreover, we shall isolate the notion of germ-like series: we say that $a \in K\left(\left(\mathbb{R}^{\leq 0}\right)\right)$ is germ-like if either ot $(a)=v_{J}(a)$, or $v_{J}(a)>1$ and ot $(a)=v_{J}(a)+1$ (see Definition 4.1). The main result of [1] can be rephrased as saying that germ-like series of order-value $\omega^{\omega^{\alpha}}$ are irreducible, while the main result of [7] is that germ-like series of order-value $\omega$ are prime. Moreover, Pommersheim and Shahriari [9] proved that germ-like series of order-value $\omega^{2}$ have a unique factorisation, and that some of them are irreducible.

By generalising an argument in [1], we shall see that germ-like series always have factorisations into irreducibles. Together with Pitteloud's result, we shall be able to prove that the factorisation into irreducibles of germ-like series of order-value at most $\omega^{3}$ must be unique. 
Theorems 4.8-4.9 All non-zero germ-like series in $K\left(\left(\mathbb{R}^{\leq 0}\right)\right)$ admit factorisations into irreducibles. Every non-zero germ-like series in $K((\mathbb{R} \leq 0))$ of order-value $\leq \omega^{3}$ admits a unique factorisation into irreducibles.

For completeness, we shall also verify that irreducible germs and series of order-value $\omega^{3}$ do exist.

Theorems 5.8-5.9 There exist irreducible germs in $K((\mathbb{R} \leq 0)) / J$ and irreducible series in $K\left(\left(\mathbb{R}^{\leq 0}\right)\right)$ of order-value $\omega^{3}$.

\section{Further remarks}

As noted before, all the known results about irreducibility and primality of generalised power series are in fact about germ-like power series. In view of this, we propose the following conjecture, which seems to be a reasonable intermediate statement between Conway's conjecture and Berarducci's conjecture.

Conjecture 1.6 Every non-zero germ-like series in $K\left(\left(\mathbb{R}^{\leq 0}\right)\right)$ admits a unique factorisation into irreducibles.

In order to treat other series that are not germ-like, we note that Lemma 4.5 and its following Corollary 4.7 suggest an alternative multiplicative order-value map whose value is the first term of the Cantor normal form of the order type, rather than the last infinite one. This has several consequences about irreducibility of general series; for instance, if $P$ is the multiplicative group of the non-zero series with finite support, it implies that the localised ring $P^{-1} K\left(\left(\mathbb{R}^{\leq 0}\right)\right)$ admit factorisation into irreducibles. Other consequences of the new order-value will be investigated in a future work.

\section{Acknowledgements}

Both authors gratefully acknowledge the support of the project FIRB2010 "New advances in the Model theory of exponentiation" RBFR10V792, of which the first author is the principal investigator. The second author was also supported by the ERC-AdG "Diophantine Problems" 267273. 


\section{Preliminaries}

\subsection{Ordinal arithmetic}

This subsection is a self-contained presentation of the classical and well-known properties of ordinal arithmetic. First, let us briefly recall how ordinals can be introduced. Two (linearly) ordered sets $X$ and $Y$ are called order similar if they are isomorphic. The order similarity is an equivalence relation and its classes are called order types.

An ordinal number is the order type of a well-ordered set, ie an ordered set with the property that any non-empty subset has a minimum. Given two ordinal numbers $\alpha, \beta$, we say that $\alpha \leq \beta$ if there are two representatives $A$ and $B$ such that $A \subseteq B$ and such that the inclusion of $A$ in $B$ is a homomorphism; we say that $\alpha<\beta$ if $\alpha \leq \beta$ and $\alpha \neq \beta$. A key observation in the theory of ordinals is that On itself is well-ordered by $\leq$. This lets us define ordinal arithmetic by induction on $\leq$ :

- the minimum ordinal in On is called zero and is denoted by 0 ;

- given an $\alpha \in \mathbf{O n}$, the successor $S(\alpha)$ of $\alpha$ is the minimum $\beta$ such that $\beta>\alpha$;

- given a set $A \subseteq \mathbf{O n}$, the supremum $\sup (A)$ is the minimum $\beta$ such that $\beta \geq \alpha$ for all $\alpha \in A$;

- sum: $\alpha+0:=\alpha, \alpha+\beta:=\sup _{\gamma<\beta}\{S(\alpha+\gamma)\}$;

- product: $\alpha \cdot 0:=0, \alpha \cdot 1:=\alpha, \alpha \cdot \beta:=\sup _{\gamma<\beta}\{\alpha \cdot \gamma+\alpha\}$;

- exponentiation: $\alpha^{0}:=1, \alpha^{\beta}:=\sup _{\gamma<\beta}\left\{\alpha^{\gamma} \cdot \alpha\right\}$.

One can easily verify that sum and product are associative, but not commutative, that the product is distributive over the sum in the second argument, and that $\alpha^{\beta+\gamma}=\alpha^{\beta} \cdot \alpha^{\gamma}$. Moreover, sum, product and exponentiation are strictly increasing and continuous in the second argument.

The finite ordinals are the ones that are represented by finite ordered sets. They can be identified with the natural numbers $0,1,2, \ldots$, where ordinal arithmetic coincides with Peano's arithmetic. The ordinals that are not zero or successors are called limit, and one can verify that $\alpha$ is a limit if and only if $\alpha \neq 0$ and $\alpha=\sup _{\beta<\alpha}\{\beta\}$. The smallest limit ordinal is called $\omega$.

The three operations admit notions of subtraction, division and logarithm. More precisely, given $\alpha \leq \beta$, there exist:

- a unique $\gamma$ such that $\alpha+\gamma=\beta$; 
- unique $\gamma, \delta$ with $\delta<\alpha$ such that $\alpha \cdot \gamma+\delta=\beta$;

- unique $\gamma, \delta, \eta$ with $\delta<\beta, \eta<\alpha$ such that $\beta^{\gamma} \cdot \delta+\eta=\alpha$.

In particular, for all $\alpha \in \mathbf{O n}$ there is a unique finite sequence $\beta_{1} \geq \beta_{2} \geq \ldots \geq \beta_{n} \geq 0$ such that

$$
\alpha=\omega^{\beta_{1}}+\ldots+\omega^{\beta_{n}} .
$$

The expression on the right-hand side is called Cantor normal form of $\alpha$. Given two ordinals in Cantor normal form, it is rather easy to calculate the Cantor normal form of their sum and product, using associativity, distributivity in the second argument and the following rules:

- if $\alpha<\beta, \omega^{\alpha}+\omega^{\beta}=\omega^{\beta}$;

- if $\alpha=\omega^{\beta_{1}}+\ldots+\omega^{\beta_{n}}$ is in Cantor normal form and $\gamma>0$, then $\alpha \cdot \omega^{\gamma}=\omega^{\beta_{1}+\gamma}$.

Finally, we recall that On also admits different commutative operations called Hessenberg's natural sum $\oplus$ and natural product $\odot$. These can be defined rather easily using the Cantor normal form. Given $\alpha=\omega^{\gamma_{1}}+\omega^{\gamma_{2}}+\ldots+\omega^{\gamma_{n}}$ and $\beta=\omega^{\gamma_{n+1}}+\omega^{\gamma_{n+2}}+\ldots+\omega^{\gamma_{n+m}}$ in Cantor normal form, the natural sum $\alpha \oplus \beta$ is defined as $\alpha \oplus \beta:=\omega^{\gamma_{\pi(1)}}+\omega^{\gamma_{\pi(2)}}+\ldots+\omega^{\gamma_{\pi(n+m)}}$, where $\pi$ is a permutation of the integers $1, \ldots, n+m$ such that $\gamma_{\pi(1)} \geq \ldots \geq \gamma_{\pi(n+m)}$, and the natural product is defined by $\alpha \odot \beta:=\bigoplus_{1 \leq i \leq n} \bigoplus_{n+1 \leq j \leq k+m} \omega^{\gamma_{i} \oplus \gamma_{j}}$.

\subsection{Order-value}

We now recall the definition and the basic properties of the order-value map introduced by Berarducci in [1].

Given $a \in K\left(\left(\mathbb{R}^{\leq 0}\right)\right)$, we let $\operatorname{ot}(a)$ be the order type of support $S_{a}$ of $a$ (recall that the support of $a$ is a well-ordered subset of $\mathbb{R}$, hence ot $(a)$ is a countable ordinal). One can verify that given two series $a, b \in K\left(\left(\mathbb{R}^{\leq 0}\right)\right)$ we have:

- $\operatorname{ot}(a+b) \leq \operatorname{ot}(a) \oplus \operatorname{ot}(b)$

- $\operatorname{ot}(a \cdot b) \leq \operatorname{ot}(a) \odot \operatorname{ot}(b)$.

However, the above inequalities may well be strict for certain values of $a$ and $b$. In order to get a better algebraic behaviour, Berarducci introduced the so called order-value $v_{J}: K\left(\left(\mathbb{R}^{\leq 0}\right)\right) \rightarrow \mathbf{O n}$ by considering only the 'tail' of the support. 
Definition 2.1 We let $J$ be the ideal of $K\left(\left(\mathbb{R}^{\leq 0}\right)\right)$ generated by the set of monomials $\left\{t^{\gamma}: \gamma \in G^{<0}\right\}$. For every $a \in K\left(\left(\mathbb{R}^{\leq 0}\right)\right)$, we call the germ of $a$ the coset $a+J \in$ $K((\mathbb{R} \leq 0)) / J$.

Remark 2.2 The ideal $J$ can also be defined by looking at the support: for every series $a, a \in J$ if and only if there exists $\gamma<0$ such that $S_{a} \leq \gamma$. In particular, $a+J=b+J$ if and only if there exists $\gamma<0$ such that for all $\delta \geq \gamma$, the coefficients of $t^{\delta}$ in $a$ and $b$ coincide.

Notation 2.3 Let $V \subseteq K\left(\left(\mathbb{R}^{\leq 0}\right)\right)$ be any $K$-vector space. Then, let us write, for $a, b \in K\left(\left(\mathbb{R}^{\leq 0}\right)\right), a \equiv b \bmod V$ if $a-b \in V$.

Definition 2.4 The order-value $v_{J}: K((\mathbb{R} \leq 0)) \rightarrow$ On is defined by:

$$
v_{J}(a):= \begin{cases}0 & \text { if } a \in J, \\ 1 & \text { if } a \notin J \text { and } a \in J+K, \\ \min \{\operatorname{ot}(b): b \equiv a \quad \bmod J+K\} & \text { otherwise. }\end{cases}
$$

Remark 2.5 Suppose that $a$ is not in $J+K$ and write ot $(a)=\omega^{\alpha_{1}}+\ldots+\omega^{\alpha_{n}}$ in Cantor normal form. Then $v_{J}(a)$ is precisely the last infinite term of the Cantor normal form of ot $(a)$ (which is either $\omega^{\alpha_{n-1}}$ or $\omega^{\alpha_{n}}$, depending on whether $0 \in S_{a}$ or $0 \notin S_{a}$ ). Note in particular that the order-value takes only values of the form $\omega^{\alpha}$ or 0 .

Furthermore, since $a \equiv b \bmod J$ implies $v_{J}(a)=v_{J}(b)$, the map $v_{J}$ induces an analogous order-value $\bar{v}_{J}: K((\mathbb{R} \leq 0)) / J \rightarrow$ On by defining $\bar{v}_{J}(a+J):=v_{J}(a)$. With a slight abuse of notation, we will use the symbol $v_{J}$ for both $v_{J}$ and $\bar{v}_{J}$.

The key and difficult result of [1] is that the function $v_{J}$ is multiplicative.

Proposition 2.6 ([1, Lemma 5.5 and Theorem 9.7]) Let $a, b \in K\left(\left(\mathbb{R}^{\leq 0}\right)\right)$. Then:

(1) $v_{J}(a+b) \leq \max \left\{v_{J}(a), v_{J}(b)\right\}$, with equality if $v_{J}(a) \neq v_{J}(b)$,

(2) $v_{J}(a b)=v_{J}(a) \odot v_{J}(b)$ (multiplicative property).

The multiplicative property is the crucial ingredient that leads to the main results in [1]. For instance, it implies the following.

Proposition 2.7 ([1, Corollary 9.8]) The ideal $J$ of $K\left(\left(\mathbb{R}^{\leq 0}\right)\right)$ is prime.

Proof Note that $a \in J$ if and only if $v_{J}(a)=0$. It follows that for all $a, b \in K((\mathbb{R} \leq 0))$, if the product $a b$ is in $J$, that is, $v_{J}(a b)=0$, then $v_{J}(a) \odot v_{J}(b)=0$, which implies $v_{J}(a)=0$ or $v_{J}(b)=0$, hence $a \in J$ or $b \in J$. 
Finally, we recall some additional notions and results from [1].

Definition 2.8 Given $a=\sum_{\beta} a_{\beta} t^{\beta} \in K\left(\left(\mathbb{R}^{\leq 0}\right)\right)$ and $\gamma \in \mathbb{R} \leq 0$, we define:

- the truncation of $a$ at $\gamma$ is $a_{\mid \gamma}:=\sum_{\beta \leq \gamma} a_{\beta} t^{\beta}$,

- the translated truncation of $a$ at $\gamma$ is $a^{\mid \gamma}:=t^{-\gamma} a_{\mid \gamma}$.

The equivalence class $a^{\mid \gamma}+J$ is the germ of $a$ at $\gamma$.

It turns out that translated truncations behave like a sort of 'generalised coefficients', as they satisfy the following equation.

Proposition 2.9 ([1, Lemma 7.5(2)]) For all $a, b \in K\left(\left(\mathbb{R}^{\leq 0}\right)\right)$ and $\gamma \in \mathbb{R} \leq 0$ we have:

$$
(a b)^{\mid \gamma} \equiv \sum_{\delta+\varepsilon=\gamma} a^{\mid \delta} b^{\mid \varepsilon} \quad \bmod J \quad \text { (convolution formula). }
$$

\section{Primality in $K\left(\left(\mathbb{R}^{\leq 0}\right)\right) / J$}

Pitteloud [7] proved that the series of order type $\omega$ or $\omega+1$ and order-value $\omega$ are prime. In this section, we adopt the same strategy to prove that every germ $a \in K((\mathbb{R} \leq 0)) / J$ of order-value $\omega$ is prime.

Following [7, p. 1209], we introduce some additional $K$-vector spaces.

Definition 3.1 ([7, p. 1209]) For $\alpha \in \mathbf{O n}$, let $J_{\omega^{\alpha}}$ be the $K$-vector space $J_{\omega^{\alpha}}:=$ $\left\{a \in K\left(\left(\mathbb{R}^{\leq 0}\right)\right): v_{J}(a)<\omega^{\alpha}\right\}$. Moreover, we write $b \mid a \bmod J_{\omega^{\alpha}}$ if there exists $c \in K\left(\left(\mathbb{R}^{\leq 0}\right)\right)$ such that $a \equiv b c \bmod J_{\omega^{\alpha}}$.

For instance, $J_{\omega^{0}}=J$ and $J_{\omega^{1}}=J+K$. Note that $J_{\omega^{\alpha}}$ is just a $K$-vector space. By the multiplicative property, one can easily verify that $J_{\omega^{\alpha}}$ is closed under multiplication if and only if $\alpha=\omega^{\beta}$ for some $\beta$, and it is an ideal if and only if $\alpha=0$.

Let $a, b, c, d \in K\left(\left(\mathbb{R}^{\leq 0}\right)\right)$ satisfy $a b=c d$ and $v_{J}(a)=\operatorname{ot}(a)=\omega$. Pitteloud proved that either $a \mid c$ or $a \mid d$ in $K((\mathbb{R} \leq 0)$ ) (where $a \mid c$ means $a$ divides $c$ ) by analysing the related equation $a^{k} b=c^{l} d$ (with $k, l>0$ ). More precisely, he proved the following:

Proposition 3.2 ([7, Prop. 3.2]) Let $a, b, c, d$ in $K((\mathbb{R} \leq 0))$ be such that $v_{J}(a)=\omega$ and assume that $a^{k} b \equiv c^{l} d \bmod J_{v_{J}\left(a^{k} b\right)}$ with $k, l>0$. Then either $a \mid c \bmod J_{v_{J}(c)}$ or $a \mid d \bmod J_{v_{J}(d)}$. 
Starting from this proposition, we can prove the following result.

Theorem 3.3 All germs in $K\left(\left(\mathbb{R}^{\leq 0}\right)\right) / J$ of order-value $\omega$ are prime.

Proof Let $A=a+J, B=b+J, C=c+J$ and $D=d+J$ be non-zero germs of $K\left(\left(\mathbb{R}^{\leq 0}\right)\right) / J$ such that $v_{J}(A)=\omega$ and $A B=C D$. We claim that $A \mid C$ or $A \mid D$. We work by induction on $v_{J}(A B)$.

Note that $v_{J}(a b-c d)=v_{J}(A B-C D)=0$, so there exists $j \in J$ such that $a b=c d+j$. Since $v_{J}(j)=0<\omega=v_{J}(a) \leq v_{J}(a b)$, we have in fact $a b \equiv c d \bmod J_{v_{J}(a b)}$. By Proposition 3.2, either $a \mid c \bmod J_{v_{J}(c)}$ or $a \mid d \bmod J_{v_{J}(d)}$.

Since $C$ and $D$ have a symmetric role, we may assume to be in the former case. Then there are $e, c^{\prime} \in K((\mathbb{R} \leq 0))$ such that $c=a e+c^{\prime}$ with $v_{J}\left(c^{\prime}\right)<v_{J}(c)$. In turn, we have

$$
a b=c d+j=\left(a e+c^{\prime}\right) d+j
$$

and in particular

$$
a(b-e d)=c^{\prime} d+j
$$

Let $B^{\prime}:=(b-e d)+J, C^{\prime}:=c^{\prime}+J, E:=e+J$. The above equation means that $A B^{\prime}=C^{\prime} D$. Now note that $v_{J}\left(A B^{\prime}\right)=v_{J}\left(C^{\prime} D\right)<v_{J}(C D)=v_{J}(A B)$. Therefore, by inductive hypothesis, either $A \mid C^{\prime}$ or $A \mid D$. In the latter case, we are done. In the former case, we just recall that $C=A E+C^{\prime}$, so $A \mid C$, as desired.

Theorem 3.4 Every non-zero germ in $K\left(\left(\mathbb{R}^{\leq 0}\right)\right) / J$ of order-value $\leq \omega^{3}$ admits a unique factorisation into irreducibles.

Proof If $v_{J}(A)=\omega$, then $A$ is prime and therefore irreducible by Theorem 3.3.

If $v_{J}(A)=\omega^{2}$ or $\omega^{3}$, then $A$ is either irreducible or equal to $A=B C$ with $v_{J}(B), v_{J}(C)<$ $v_{J}(A)$. We assume to be in the latter case. Since $v_{J}(B) \odot v_{J}(C)=v_{J}(A)$, we must have that either $v_{J}(B)=\omega$ or $v_{J}(C)=\omega$; by symmetry, we may assume that $v_{J}(B)=\omega$, and in particular that $B$ is prime.

If $A$ has another factorisation into irreducibles, then $B$ must divide one of the factors, and in particular it must be equal to one of the factors up to a unit. The product of the remaining factors has either order-value $\omega^{2}$ or $\omega$; in both cases we repeat the argument and we are done. 


\section{Germ-like series}

Unfortunately, even if a series in $K((\mathbb{R}))^{\leq 0}$ has an irreducible germ, it may well be reducible (see for instance $\left.\left(t^{-1}-1\right)\left(1+\sum_{n} t^{-\frac{1}{n}}\right)\right)$. This implies that the results on germs cannot be lifted automatically to all series. On the other hand, there are some series which behave similarly enough to germs so that the same techniques can be applied to them.

Definition 4.1 We say that an $a \in K\left(\left(\mathbb{R}^{\leq 0}\right)\right)$ is germ-like if either $\operatorname{ot}(a)=v_{J}(a)$ or $v_{J}(a)>1$ and $\operatorname{ot}(a)=v_{J}(a)+1$.

We shall prove that if a product of non-zero series is germ-like, then the series themselves are germ-like. For this, we recall the following definition from [1].

Definition 4.2 Given $a \in K\left(\left(\mathbb{R}^{\leq 0}\right)\right)^{*}$, let $\alpha=\max \left\{v_{J}\left(a^{\mid \gamma}\right): \gamma \in \mathbb{R}^{\leq 0}\right\}$. The critical point $\operatorname{crit}(a)$ of $a$ is the least $\gamma \in \mathbb{R} \leq 0$ such that $v_{J}\left(a^{\mid \gamma}\right)=\alpha$. We also set $\operatorname{crit}(0):=0$.

Remark 4.3 By construction, $v_{J}\left(a^{\mid \operatorname{crit}(a)}\right)$ is equal to the first term of the Cantor normal form of the order type of $a$.

Proving that a critical point always exists is not difficult, and we refer to $[1, \S 10]$ for the relevant details.

Lemma 4.4 If $a \in K\left(\left(\mathbb{R}^{\leq 0}\right)\right)$, $a$ is germ-like if and only if $\operatorname{crit}(a)=0$.

Proof First of all, we note that if $a \in J$, then $v_{J}(a)=0$; in this case, we just note that $\operatorname{ot}(a)=0$ if and only if $a=0$, and the conclusion follows trivially. Similarly, if $a \in J+K$ but $a \notin J$, then $v_{J}(a)=1$, and clearly ot $(a)=1$ if and only if $a \in K^{*}$, proving again the conclusion.

Now assume that $a \notin J+K$. Recall that in this case $v_{J}(a)$ is the last infinite term of the Cantor normal form of ot $(a)$ (in particular, $v_{J}(a)>1$; see Remark 2.5). Therefore, $\operatorname{ot}(a)=v_{J}(a)$ holds if and only if the Cantor normal form of ot $(a)$ is $\omega^{\alpha}$ for some $\alpha \in \mathbf{O n}$. Similarly, $v_{J}(a)=\operatorname{ot}(a)+1$ holds if and only if the Cantor normal form of $\operatorname{ot}(a)$ is $\omega^{\alpha}+1$ for some $\alpha \in \mathbf{O n}$.

If ot $(a)$ is $\omega^{\alpha}$ or $\omega^{\alpha}+1$, since $a \notin J+K$, we have ot $\left(a^{\mid \gamma}\right)<\omega^{\alpha}$ for all $\gamma \in \mathbb{R}^{<0}$. In particular, $v_{J}\left(a^{\mid \gamma}\right) \leq \operatorname{ot}\left(a^{\mid \gamma}\right)<\omega^{\alpha}=v_{J}(a)$ for all $x \in \mathbb{R}^{<0}$, hence $\operatorname{crit}(a)=0$.

Otherwise, we have that the Cantor normal form of ot $(a)$ is $\omega^{\beta_{1}}+\ldots+\omega^{\beta_{k}}$ or $\omega^{\beta_{1}}+\ldots+\omega^{\beta_{k}}+1$, where $k>1$ and $\beta_{k}>0$; let $\gamma \in \mathbb{R} \leq 0$ be the minimum real number such that $\operatorname{ot}\left(a^{\mid \gamma}\right) \geq \omega^{\beta_{1}}$. Then $\gamma$ is negative, and it is easy to verify that $v_{J}\left(a^{\mid \gamma}\right)=\omega^{\beta_{1}}$ and in fact that $\gamma=\operatorname{crit}(a)$, thus $\operatorname{crit}(a)<0$. 
The following lemma is inspired by Berarducci $[1, \S 10]$.

Lemma 4.5 If $b, c$ are non-zero series of $K\left(\left(\mathbb{R}^{\leq 0}\right)\right)$, then

(1) $\operatorname{crit}(b c)=\operatorname{crit}(b)+\operatorname{crit}(c)$;

(2) $v_{J}\left((b c)^{\mid \operatorname{crit}(b c)}\right)=v_{J}\left(b^{\mid \operatorname{crit}(b)}\right) \odot v_{J}\left(c^{\mid \operatorname{crit}(c)}\right)$.

Proof We proceed as in [1, Lemma 10.4]. Let $\gamma=\operatorname{crit}(b)$ and $\delta=\operatorname{crit}(c)$. By the convolution formula, for any $\varepsilon \in \mathbb{R}^{\leq 0}$ we have

$$
(b c)^{\mid \varepsilon} \equiv \sum_{\gamma^{\prime}+\delta^{\prime}=\varepsilon} b^{\mid \gamma^{\prime}} c^{\mid \delta^{\prime}} \bmod J .
$$

So, $v_{J}\left((b c)^{\mid \varepsilon}\right) \leq \max _{\gamma^{\prime}+\delta^{\prime}=\varepsilon}\left\{v_{J}\left(b^{\mid \gamma^{\prime}}\right) \odot v_{J}\left(c^{\mid \delta^{\prime}}\right)\right\}$. It follows at once that $v_{J}\left((b c)^{\mid \varepsilon}\right) \leq$ $v_{J}\left(b^{\mid \gamma}\right) \odot v_{J}\left(c^{\mid \delta}\right)$, and if the equality is attained, then for some $\gamma^{\prime}, \delta^{\prime}$ such that $\gamma^{\prime}+\delta^{\prime}=\varepsilon$ we have $v_{J}\left(b^{\mid \gamma^{\prime}}\right)=v_{J}\left(b^{\mid \gamma}\right)$ and $v_{J}\left(c^{\mid \delta^{\prime}}\right)=v_{J}\left(c^{\mid \delta}\right)$. In particular, if the equality holds, then $\varepsilon=\gamma^{\prime}+\delta^{\prime} \geq \gamma+\delta$.

On the other hand,

$$
(b c)^{\mid \gamma+\delta} \equiv b^{\mid \gamma} c^{\mid \delta}+\sum_{\substack{\gamma^{\prime}+\delta^{\prime}=\gamma+\delta \\ \gamma^{\prime}<\gamma}} b^{\mid \gamma^{\prime}} c^{\mid \delta^{\prime}}+\sum_{\substack{\gamma^{\prime}+\delta^{\prime}=\gamma+\delta \\ \delta^{\prime}<\delta}} b^{\mid \gamma^{\prime}} c^{\mid \delta^{\prime}} \bmod J .
$$

It immediately follows that $v_{J}\left((b c)^{\mid \gamma+\delta}\right)=v_{J}\left(b^{\mid \gamma} c^{\mid \delta}\right)=v_{J}\left(b^{\mid \gamma}\right) \odot v_{J}\left(c^{\mid \delta}\right)$. Therefore, $\operatorname{crit}(b c)=\gamma+\delta$, proving both conclusions.

Corollary 4.6 Let $a, b, c \in K\left(\left(\mathbb{R}^{\leq 0}\right)\right)$ be non-zero series with $a=b c$. Then $a$ is germ-like if and only if $b$ and $c$ are germ-like.

Proof By Lemmas 4.4, 4.5, it suffices to note that $\operatorname{crit}(a)=\operatorname{crit}(b)+\operatorname{crit}(c)$, so $\operatorname{crit}(a)=0$ if and only if $\operatorname{crit}(b)=\operatorname{crit}(c)=0$.

Corollary 4.7 The function $a \mapsto v_{J}\left(a^{\mid \operatorname{crit}(a)}\right)$ is multiplicative.

Theorem 4.8 All non-zero germ-like series in $K\left(\left(\mathbb{R}^{\leq 0}\right)\right)$ admit factorisations into irreducibles.

Proof We prove the conclusion by induction on $v_{J}(a)$. If $v_{J}(a)=1$, then we must have $\operatorname{ot}(a)=v_{J}(a)=1$, which implies that $a \in K$, and the conclusion follows trivially. If $v_{J}(a)>1$, then either $a$ is irreducible, in which case we are done, or $a=b c$ for some $b, c \in K((\mathbb{R} \leq 0)) \backslash K$. By Corollary 4.6, $b$ and $c$ are germ-like. If $v_{J}(b)=1$, 
then by the previous argument we have $b \in K$, a contradiction, hence $v_{J}(b)>1$; similarly, we deduce that $v_{J}(c)>1$ as well. By the multiplicative property, it follows that $v_{J}(b), v_{J}(c)<v_{J}(a)$; by induction, $b$ and $c$ have a factorisation into irreducibles, and we are done.

Theorem 4.9 Every non-zero germ-like series in $K((\mathbb{R} \leq 0))$ of order-value $\leq \omega^{3}$ admits a unique factorisation into irreducibles.

Proof Let $a \in K\left(\left(\mathbb{R}^{\leq 0}\right)\right)$ be a germ-like series of order value $v_{J}(a) \leq \omega^{3}$. Suppose that $a$ has a non-trivial factorisation $a=b c$. By Corollary 4.6, $b$ and $c$ are germ-like. By the multiplicative property, and possibly by swapping $b$ and $c$, we may assume $v_{J}(b) \leq \omega$.

If $v_{J}(b)=1$, then ot $(b)=1$, hence $b \in K$, contradicting the hypothesis that the factorisation is non-trivial. It follows that $v_{J}(b)=\omega$. Since $b$ is germ-like, we have $\operatorname{ot}(b)=\omega \operatorname{or} \operatorname{ot}(b)=\omega+1$, in which case $b$ is prime by Pitteloud [7, Theorem 3.3]. Once we have a prime factor, one can deduce easily that the factorisation is unique, as in the proof of Theorem 3.4.

Moreover, we observe that irreducible germs do lift to irreducible germ-like series.

Proposition 4.10 Let $a \in K((\mathbb{R} \leq 0))$ be germ-like. If the germ $a+J$ of $a$ is irreducible in $K\left(\left(\mathbb{R}^{\leq 0}\right)\right) / J$, then $a$ is irreducible in $K\left(\left(\mathbb{R}^{\leq 0}\right)\right)$.

Proof Suppose that $a=b c$ and that $a+J$ is irreducible. It immediately follows that one of $b+J$ or $c+J$ is a unit in $K\left(\left(\mathbb{R}^{\leq 0}\right)\right)$, say $b+J$. By the multiplicative property, we have that $v_{J}(b)=1$. Since $b$ is germ-like it follows that $\operatorname{ot}(b)=1$, which implies that $b \in K^{*}$, hence that $b$ is a unit in $K\left(\left(\mathbb{R}^{\leq 0}\right)\right)$, as desired.

Remark 4.11 In general, the converse does not hold. Indeed, let $a, b$ be two germ-like series of order-value $\omega$. By Pommersheim and Shahriari [9, Corollary 3.4], for all $\gamma \in \mathbb{R}^{<0}$ except at most countably many, $a b+t^{\gamma}$ is irreducible, while of course its germ $a b+t^{\gamma}+J=a b+J=(a+J)(b+J)$ is reducible.

\section{Irreducibles of order-value $\omega^{3}$}

We conclude by showing that there are several irreducible elements in both $K\left(\left(\mathbb{R}^{\leq 0}\right)\right)$ and $K\left(\left(\mathbb{R}^{\leq 0}\right)\right) / J$ of order-value $\omega^{3}$. We follow a strategy similar to the one of [9]. 
Lemma 5.1 Let $a, b \in K\left(\left(\mathbb{R}^{\leq 0}\right)\right)$. If $a \equiv b \bmod J_{\omega^{\alpha+1}}$, then for all $\gamma \in \mathbb{R}^{<0}$ sufficiently close to 0 we have $a^{\mid \gamma} \equiv b^{\mid \gamma} \bmod J_{\omega^{\alpha}}$.

Proof Let $c=a-b$, so that $v_{J}(c)<\omega^{\alpha+1}$. Note that for all $\gamma<0$ sufficiently close to 0 we have $v_{J}\left(c^{\mid \gamma}\right)<v_{J}(c)$, because then the tail of $c^{\mid \gamma}$ is a proper (translated) truncation of the tail of $c$. In particular, $v_{J}\left(c^{\mid \gamma}\right)<\omega^{\alpha}=v_{J}(c)$. Since $a^{\mid \gamma}-b^{\mid \gamma}=c^{\mid \gamma}$, we then have $a^{\mid \gamma} \equiv b^{\mid \gamma} \bmod J_{\omega^{\alpha}}$.

Given $a \in K\left(\left(\mathbb{R}^{\leq 0}\right)\right)$ and $\gamma \in \mathbb{R}^{<0}$, we let $V_{\gamma}(a)$ be the $K$-linear space generated by all the germs of $a$ between $\gamma$ and 0 , modulo $J+K$; formally,

$$
V_{\gamma}(a):=\operatorname{span}_{K}\left\{a^{\mid \delta}+J+K: \gamma<\delta<0\right\} \subseteq K((\mathbb{R} \leq 0)) /(J+K) .
$$

In particular, $V_{\gamma}(a)$ contains all the germs $a^{\mid \delta}+J$, for $\gamma<\delta<0$, modulo the subspace $J+K$.

Note that the spaces $V_{\gamma}(a)$ form a directed system, as clearly $V_{\gamma}(a) \supseteq V_{\gamma^{\prime}}(a)$ for $\gamma<\gamma^{\prime}$. We let $V(a)$ be their intersection for $\gamma \in \mathbb{R}^{<0}$ :

$$
V(a):=\bigcap_{\gamma<0} V_{\gamma}(a)
$$

The spaces $V(a)$ contains, for instance, the germs $b+J$ (modulo $J+K$ ) that appear repeatedly as $b+J=a^{\mid \delta}+J$ for $\delta$ approaching 0 .

Remark 5.2 If $a \equiv b \bmod J$, then $V_{\gamma}(a)=V_{\gamma}(b)$ for all $\gamma<0$ sufficiently close to 0 , so in fact $V(a)=V(b)$. In particular, it is well defined to write $V(a+J):=V(a)$ for any given germ $a+J$.

Proposition 5.3 Let $a, b, c \in K\left(\left(\mathbb{R}^{\leq 0}\right)\right)$ be such that $a \equiv b c \bmod J_{\omega^{2}}$ and $v_{J}(b)=$ $v_{J}(c)=\omega$. Then $\operatorname{dim}(V(a)) \leq 2$.

Proof By Lemma 5.1, for all $\gamma<0$ sufficiently close to 0 we have $a^{\mid \gamma} \equiv(b c)^{\mid \gamma}$ $\bmod J_{\omega}=J+K$. By the convolution formula, $(b c)^{\mid \gamma} \equiv \sum_{\delta+\varepsilon=\gamma} b^{\mid \delta} c^{\mid \varepsilon} \bmod J$.

Note that when $\delta<0$ is sufficiently close to 0 , we have $b^{\mid \delta}, c^{\mid \delta} \in J+K$. This in particular implies that if $\gamma$ is sufficiently close to 0 , then $(b c)^{\mid \gamma} \equiv b^{\mid \gamma} c+b c^{\mid \gamma}$ $\bmod J_{\omega}=J+K$. Moreover, we must have $b^{\mid \gamma}, c^{\mid \gamma} \in J+K$; in other words, $b^{\mid \gamma}=k_{b}+j_{b}, c^{\mid \gamma}=k_{c}+j_{c}$ for some $k_{b}, k_{c} \in K$ and $j_{b}, j_{c} \in J$. It follows that when $\gamma<0$ is sufficiently close to 0 , we have

$$
a^{\gamma} \equiv k_{b} c+k_{c} b+j_{b} c+j_{c} b \equiv k_{b} c+k_{c} b \quad \bmod J+K .
$$

Therefore, $V(a)$ is generated as $K$-vector space by $b+J+K$ and $c+J+K$, hence $\operatorname{dim}(V(a)) \leq 2$. 
In order to find a sufficient criterion for irreducibility of series of order-value $\omega^{3}$, we picture a series $a \in K\left(\left(\mathbb{R}^{\leq 0}\right)\right)$ of order-value $\omega^{\alpha+1}$ as if it were a series of order-value $\omega$ with coefficients that are themselves series of order-value $\omega^{\alpha}$. In other words, we describe $a$ as the sum of $\omega$ series of order-value $\omega^{\alpha}$.

Definition 5.4 Let $\alpha \in$ On and $a \in K((\mathbb{R} \leq 0))$ be such that $v_{J}(a)=\omega^{\alpha+1}$. We say that $\gamma \in S_{a}$ is a big point of $a$ if $v_{J}\left(a^{\mid \gamma}\right)=\omega^{\alpha}$.

Remark 5.5 By construction, the big points of a series must accumulate to 0 .

We can use big points to give the following sufficient criterion for the irreducibility of a series in $K\left(\left(\mathbb{R}^{\leq 0}\right)\right)$ of order-value $\omega^{3}$.

Proposition 5.6 Let $a, b, c \in K((\mathbb{R} \leq 0))$ be such that $a \equiv b c \bmod J, v_{J}(b)=\omega$ and $v_{J}(c)=\omega^{2}$. Let $\gamma, \delta$ be two big points of a sufficiently close to 0 . Then there exist $r, s \in K$, not both zero, such that $\operatorname{dim}\left(V\left(r a^{\mid \gamma}+s a^{\mid \delta}\right)\right) \leq 2$.

Proof By the convolution formula, when $\gamma, \delta$ are sufficiently close to 0 we have

$$
a^{\mid \gamma} \equiv b^{\mid \gamma} c+b c^{\mid \gamma} \quad \bmod J_{\omega^{2}}, \quad a^{\mid \delta} \equiv b^{\mid \delta} c+b c^{\mid \delta} \quad \bmod J_{\omega^{2}} .
$$

If $b^{\mid \gamma}=b^{\mid \delta}=0$, then we can take $r=1, s=0$ and apply Proposition 5.3 to obtain the conclusion. Otherwise, we have

$$
b^{\mid \delta} a^{\mid \gamma}-b^{\mid \gamma} a^{\mid \delta} \equiv b\left(b^{\mid \delta} c^{\mid \gamma}-b^{\mid \gamma} c^{\mid \delta}\right) \quad \bmod J_{\omega^{2}} .
$$

Let $r:=b^{\mid \delta}, s:=-b^{\mid \gamma}$. If $v_{J}\left(r a^{\mid \gamma}+s a^{\mid \delta}\right) \leq \omega$, then $V\left(r a^{\mid \gamma}+s a^{\mid \delta}\right)=0$, and we are done. Otherwise, by the previous equation and Proposition 5.3 we get $\operatorname{dim}\left(V\left(r a^{\mid \gamma}+s a^{\mid \delta}\right)\right) \leq 2$, as desired.

It is now not difficult to construct several series $a \in K\left(\left(\mathbb{R}^{\leq 0}\right)\right)$ of order-value $\omega^{3}$ such that the condition

$$
\operatorname{dim}\left(V\left(r a^{\mid \gamma}+s a^{\mid \delta}\right)\right)>2
$$

is satisfied for all $r, s \in K$ not both zero and for all distinct big points $\gamma \neq \delta$ of $a$. In particular, their corresponding germs $a+J$ are all irreducible. Indeed, we observe the following.

Lemma 5.7 Let $\left(a_{i} \in K\left(\left(\mathbb{R}^{\leq 0}\right)\right): i \in \mathbb{N}\right)$ be a sequence of series of order type $\omega^{\alpha}$, and let $\left(\gamma_{i} \in \mathbb{R}^{<0}: i \in \mathbb{N}\right)$ be a strictly increasing sequence of negative real numbers such that $\lim _{i \rightarrow \infty} \gamma_{i}=0$. Then there exists $a \in K((\mathbb{R} \leq 0))$ with $v_{J}(a)=\omega^{\alpha+1}$ whose big points are the exponents $\gamma_{i}$ and such that $a^{\mid \gamma_{i}} \equiv a_{i} \bmod J$ for all $i \in \mathbb{N}$. 
Proof First of all, we may assume that $S_{a_{i+1}}>\gamma_{i}-\gamma_{i+1}$ for all $i \in \mathbb{N}$. Indeed, if this is not the case, it suffices to replace $a_{i+1}$ with the series $a_{i+1}-\left(a_{i+1}\right)_{\mid \gamma_{i}-\gamma_{i+1}} \equiv a_{i+1}$ mod $J$. In particular, by construction, the supports of the series $t^{\gamma_{i}} a_{i}$ do not overlap. This implies that the following infinite sum is well defined:

$$
a:=\sum_{i \in \mathbb{N}} t^{\gamma_{i}} a_{i}
$$

Since $S_{t}^{\gamma_{i+1} a_{i+1}}>\gamma_{i}$, we then have

$$
a^{\mid \gamma_{i}}=\sum_{j \leq i} t^{\gamma_{j}-\gamma_{i}} a_{j} \equiv a_{i} \quad \bmod J
$$

and the exponents $\gamma_{i}$ are exactly the big points of $a$.

Theorem 5.8 There exist irreducible germs in $K((\mathbb{R} \leq 0)) / J$ of order-value $\omega^{3}$.

Proof Let $\Omega$ be any countable set of series of order-value $\omega$ with pairwise disjoint supports. Clearly, $\Omega$ is a $K$-linearly independent set. Moreover, it is $K$-linearly independent even modulo the vector space $J+K$. Let $a_{i}$, for $i \in \mathbb{N}$, be some enumeration of $\Omega$, and take a strictly increasing sequence $\left(\gamma_{i} \in \mathbb{R}^{<0}: i \in \mathbb{N}\right)$ such that $\lim _{i \rightarrow \infty} \gamma_{i}=0$.

By Lemma 5.7, there are series $b_{i}$ of order-value $\omega^{2}$ such that

$$
b_{i}^{\gamma_{3 h+k}} \equiv a_{3 i+k} \quad \bmod J .
$$

for all $i, h \in \mathbb{N}$ and $k \in\{0,1,2\}$. By Lemma 5.7 again, there exists $c$ of order-value $\omega^{3}$ such that

$$
c^{\mid \gamma_{i}} \equiv b_{i} \quad \bmod J
$$

and whose big points are the $\gamma_{i}$ 's.

Take any $r, s \in K$ not both zero and any two distinct $\gamma_{i}, \gamma_{j}$. By construction, $r c^{\mid \gamma_{i}}+$ $s c^{\mid \gamma_{j}} \equiv r b_{i}+s b_{j} \bmod J$. Note moreover that for any $\gamma_{3 h+k}$,

$$
\left(r b_{i}+s b_{j}\right)^{\mid \gamma_{3 h+k}}=r b_{i}^{\mid \gamma_{3 h+k}}+s b_{j}^{\mid \gamma_{3 h+k}} \equiv r a_{3 i+k}+s a_{3 j+k} \quad \bmod J .
$$

It follows at once that

$$
\begin{aligned}
& V\left(r c^{\mid \gamma_{i}}+s c^{\mid \gamma_{j}}\right)=V\left(r b_{i}+s b_{j}\right) \supseteq \\
& \quad\left\{r a_{3 i}+s a_{3 j}+J+K, r a_{3 i+1}+s a_{3 j+1}+J+K, r a_{3 i+2}+s a_{3 j+2}+J+K\right\} .
\end{aligned}
$$

By elementary linear algebra, it follows that for all $i \neq j$ we have

$$
\operatorname{dim}\left(V\left(r c^{\mid \gamma_{i}}+s c^{\mid \gamma_{j}}\right)\right) \geq 3
$$

By Proposition 5.6, it follows that $c+J$ is irreducible. 
Theorem 5.9 There exist irreducible series in $K((\mathbb{R} \leq 0))$ of order-value $\omega^{3}$.

Proof By Theorem 5.8, there exist series $c$ of order-value $\omega^{3}$ such that $c+J$ is irreducible. Up to replacing $c$ with $c-c_{\mid \gamma}$ for a suitable $\gamma \in \mathbb{R}^{<0}$, we may directly assume that $c$ is germ-like. By Proposition 4.10, $c$ is irreducible.

\section{References}

[1] A Berarducci, Factorization in Generalized Power Series, Transactions of the American Mathematical Society 352 (2000) 553-577; doi: 10.1090/S0002-9947-99-02172-8

[2] D Biljakovic, M Kochetov, S Kuhlmann, Primes and irreducibles in truncation integer parts of real closed fields, from: "Logic in Tehran", Lect. Notes Log. 26, Assoc. Symbol. Logic, La Jolla, CA (2006) 42-64

[3] J H Conway, On number and Games, London Mathematical Society Monographs, Academic Press, London (1976)

[4] A Gleyzal, Transfinite Real Numbers, Proceedings of the National Academy of Sciences of the United States of America 23 (1937) 581-587; doi: 10.1073/pnas.23.11.581

[5] H Hahn, Über die nichtarchimedischen Größensysteme, from: "Hans Hahn Gesammelte Abhandlungen Band 1/Hans Hahn Collected Works Volume 1", volume 116, Springer Vienna, Vienna (1995) 445-499; doi: 10.1007/978-3-7091-6601-7_18

[6] MH Mourgues, J-P Ressayre, Every Real Closed Field has an Integer Part, The Journal of Symbolic Logic 58 (1993) 641-647; doi: 10.2307/2275224

[7] D Pitteloud, Existence of prime elements in rings of generalized power series, Journal of Symbolic Logic 66 (2001) 1206-1216; doi: 10.2307/2695102

[8] D Pitteloud, Algebraic properties of rings of generalized power series, Annals of Pure and Applied Logic 116 (2002) 39-66; doi: 10.1016/S0168-0072(01)00099-9

[9] J Pommersheim, S Shahriari, Unique factorization in generalized power series rings, Proceedings of the American Mathematical Society 134 (2005) 1277-1287; doi: 10.1090/S0002-9939-05-08162-1

School of Science and Technology, Mathematics Division, University of Camerino, Via Madonna delle Carceri 9, I-62032 Camerino, Italy

Scuola Normale Superiore, Faculty of Sciences, Piazza dei Cavalieri 7, I-56126 Pisa, Italy

sonia.linnocente@unicam.it, v.l.mantova@leeds.ac.uk

Received: 15 December 2015 Revised: 31 January 2017 